\title{
Norms in Translation: A Case Study of Telugu
}

\section{H. Lakshmi}

\section{$\underline{\text { Abstract }}$}

The concept of 'norms' was introduced into Translation Studies by Gideon Toury, the pioneer of Descriptive Translation Studies, in 1978, to refer to general values or ideas shared by a community. It is the norms that inform the decision making process of the translation as they function as the socio-cultural constraints specific to a culture, society and time and become prescriptive in nature. The translators as members of a given socio cultural, historical and temporal context would know the norms of translation behaviour that are in operation in their contexts and try to observe them in their translation. The present paper makes an attempt to examine and analyse some paratexts that accompanied translated texts in Telugu to understand the norms of translation behaviour that are in vogue in Telugu and to know the predominant trends in translation that play a role in determining what a good translation is or should be. This study also brings to light to some extent translation discourse in Telugu.

Keywords: Norms, Telugu, translation

\section{Discussion:}

Gideon Toury, the pioneer of Descriptive Translation Studies, has introduced the concept called "Norms" into Translation Studies in 1978 with the publication of his article entitled, 'The Nature and Role of Norms in Translation'. Continuing and building on the 
Polysystem theory proposed by his teacher Itamar Evan- Zohar that argues that translated literature is a part of the social, cultural, historical and literary system of the target language and thus forms a system within the polysystem of the target language and hence cannot be studied in isolation, Toury aims to distinguish trends of translation behaviour and the factors that influence the translator's decision making process by invoking the concept of norms. He defines norms as, "the translation of general values or ideas shared by a community-as to what is right or wrong, adequate or inadequate-into performance instructions appropriate for and applicable to particular situations" (Toury,1995,p.55).

It is the norms that inform the decision making process of the translation as they function as the sociocultural constraints specific to a culture, society and time and become prescriptive in nature. The concept of norms is employed in the analysis of a translation product as it is the norms that govern the nature of the translation equivalence manifested therein. The translators as members of a given socio cultural, historical and temporal context would be well aware of the norms of translation behaviour that are in operation in their contexts and follow them in their translation. Now the question is how do we arrive at the norms that are in operation in the translation of a particular text? Toury proposes that the norms can be reconstructed from the following two types of sources: a) from the comparative analysis of the ST and TT, by examining the correspondences between the two which helps us understand the translation process/method/strategy adopted by the translator (this is a typical product oriented study that throws light on the process behind its making); b) from the explicit statements made about norms by translators, publishers, reviewers and the like. This is nothing but the examination of the paratextual elements of a 
translated text. Here Toury also adds a word of caution-such statements (paratextual) may be biased in favour of the role played by the informants in the socio-cultural system. This is quite understandable as we also know that sometimes there will not be any correlation between what the translators state in their introduction about their own translation strategy and what they actually do.

The study of paratextual elements, however, proves very useful in understanding not only the norms that are in operation in a given society at a given time but also various other aspects related to the act of translation in general (Genette, 1991). Generally, in translated texts, we find paratexts like introduction either by the translator or some expert, foreword by some eminent personality in the field, a note by the publisher and the translator's note or introduction by the translator. These paratexts help us not only to understand the text better by situating it in a particular socio-cultural, historical and temporal context but also to know what is considered to be an acceptable translation by the target community in general. The paratexts also reveal what the approach of the experts or critics and the translators is towards a translation and what they consider to be a good translation which would in turn reflect also the reader's expectations of what an acceptable translation is in the target culture. The remarks made by such people make it clear to us the criteria or parameters used in translation evaluation/translation criticism and translation review which are based on translation norms that are in vogue in the given society at the given time. These paratexts thus help us understand the norms of translation behaviour that are in operation in the target culture that find voice in the statements issued by the experts and the translators alike. 
In this context let us look at the norms proposed by Chesterman (1993) as well. Chesterman on the basis of the work done by Toury and Hermans proposes two kinds of norms. These are a) Expectancy norms and b) Professional norms. Expectancy norms refer to what the target language community expects a translation to look like regarding grammaticality, acceptability, appropriateness, style, textuality, preferred conventions of form or discourse and the like (Chesterman,1993,p.17). And the professional norms govern the accepted methods and strategies of the translation process.

Against this backdrop, let us now examine some paratexts that accompany translated texts in Telugu to understand the prevailing norms of translation behaviour in the Telugu context and to know the predominant trends in translation that determine what a good translation is or should be. As understood, translations are shaped by the norms of the target culture since the translators as members of the target community prioritize these norms in their translations and shape their translations accordingly. Even the selection of the source text to be translated and its production are determined by the third factor- the reception as the reading habit of the target audience plays a key role in informing the other two and thus plays a major role in shaping the translation.

An attempt is thus made in this study to examine literary translations made into Telugu from other languages in order to cull out paratexts and analyse them to arrive at some understanding of translation norms that operate in Telugu translation sphere. On the basis of the analysis of the selected paratexts the following observations are made:

I. The Telugu translators have tremendous respect and high regard for the source authors and their writings that they have chosen to translate into Telugu, whether they are 
epics, kavyas or plays from Sanskrit or literary texts of any genre from English or any other Indian language or a foreign language. This seems to be one of the preconditions for the selection of the text for translation. It could also be taken as a sign of their modesty and humility.

Let us now look at some of the statements made by the translators:

a) Prof. G.N. Reddy in his foreword to the translation of the Gitanjali by Sri Anjaneya Sharma states the following:

“అనువాదకునికి మూలగ్రంథం మీద అభిమానం, తద్గంధకర్త మీద భక్తి వుండటమీకాకుండ మూలగ్రంథకర్తకున్న ప్రవృత్తికి తన ప్రవృత్తికి సాదృశ్యం వుండాలి. అపుడే అనువాదంలో కొంత నిజాయితీ వుంటుంది. అనువాదరచన సార్థకమవుతుంది. డా. ఆంజనేయశర్మగారు ఆధ్యాత్మికమైన ప్రవృత్తి, బావనగలవారు కాబ్టటే వారిని గీతాంజలి ఆకర్షించింది".

("The translator should have not only great love for the source text and deep devotion towards the source author but also needs to have empathy with the source writer. Only then the translation appears genuine and the purpose of the translation would be fulfilled. Since Dr. Anjaneya Sharma has got spiritual disposition and temperament, he got attracted towards the Gitanjali”).

b) Lakshmikanta Mohan who has translated almost all of Shakespeare's works into Telugu makes the following remark regarding Shakespeare's writings: 
H. Lakshmi

షేక్స్పియర్ రచనలు మల్లెపువ్వులాగా కోమలమైనట్టివి, సుగంధ పూరితమైనట్టివి. ఆ కోమలత్వం, ఆ సుగంధం యెక్కడ చెడిపోతాయోననే భయంతోనే నేను ఈ నాటకాన్ని అనువాదం జేశాను.

(Shakespeare's writings are tender like jasmines and filled with great fragrance. I have translated this play with the fear of spoiling that tenderness and fragrance.)

c) Rayaprolu Subbarao in his translator's introduction to his Telugu translation-Ravindra Vyasavali praises the greatness of Tagore's writings as follows:

రవీంద్రుని రచన గద్యలో పద్యములో పాటలో సంవాదములో వివిధ రుచి రూపాలతో ఒదిగి ఒప్పి - ఋతువులలో ప్రకృతిలాగా -ప్రసరించింది. ఆయన శైలిలో భావనా - బావమూ, భాషా భణితీ అపూర్వంగా భాసిస్తవి. ప్రజ్ఞా - ప్రతిభా నర్తనం చేస్తవి. సృజనా - కల్పనా ఎంత నవీనంగా ఉంటవో రచన అంత నిరంకుశలంగా ప్రవహిస్తుంది. పైగా, ఎక్కడ పట్టినా, దరహాస పరిహాసములు చెమ్మచెక్కలాడుతున్నట్లు హాస్యలాలన విభ్రమిస్తుంది. ఈ నిర్వహణకోసం ఆయన సమకాల సజీవమైన సమాజభాషితమును జంకుకొంకులు లేకుండా వాడుకొన్నాడు. 'నియతికృత నియమరహితాం' అనే నిర్వచనం రవీంద్ర సాహిత్యానికి చక్కగా సరిపోతుంది.

ఇంతేకాదు, రవీంద్రుడు వస్తుతః గాంధర్వ పుత్రుడు. అంతర్విపంచి తంత్రులు ఎప్పుడూ మ్రూగుతునే వుంటవి. శ్రుతి స్వరలయలు - 
ధాతువులలో రక్తధారలలాగా - సర్వదా స్పందిస్తూనే వుంటవి. నిద్రలో సంగతి తెలియదు. పిండిన పాలమీద బుడగలలాగా శబ్దాలు పైకి తేలి మళ్ళీ రసీభవిస్తవి. ఇది ఆయన శైలి. లీలామయి భగవతిప్రకృతి. స్వరమయి గీతి - భావమయి కవిత - చేష్టామయి భణితి - అని ఆకాంక్షించుకోవాలె ఆయన ్యోతిర్మయ వాజ్మయంలో

(The way in which the nature smoothly turns into different seasons. Tagore's creative writing gets embedded and settled down into prose, poetry, song and dialogue with all hues and forms. In his style the thought and expression, the language and sense shine amazingly and his skill and wisdom dance beautifully. His writing flows as smoothly as the novelty of his innovation and creation. On top of it, it amuses us with its interplay of wit and humor everywhere. To manage this, he used the contemporary live social dialogue without any hesitation. The definition, 'rulebound, un-bound' is perfectly suitable for Tagore's literature.

This is not all. Tagore is by nature the son of God of Music. The strings would always keep playing. The rhythmic melody acts upon forever -like the flow of blood in veins. It is not known what happens when he sleeps! Like the foam that springs up in the pot when you milk (a cow), the sounds spring up and then settle down into great melody. That is his style. Magic in nature. Melody in song-emotion in poem - this is how we need to approach his luminous literature.) 
d) Dr. R. Anantha Padmanabha Rao in the introduction to his Telugu translation of Mulkraj Anand's Morning Face remarks as follows:

గంగాఝరీ వేగంతో సమానమైన శైలి ఆనంద్గారిది. ఆయన ఆంగ్లవాక్య విన్యాసం, భాషాపటిమ చదువరులను ఆకట్టుకొని ఆసాంతం చదివింపజేస్తాయి. దాని అనువాదం కత్తిమీద సాము వంటిది. మూలంలో భావానికి లోపం రాకుండా, తెలుగునుడికారం సొంపులకు వొదిగేలా నేను అనువాదం సాగించాను.

(Mr. Anand's style of writing is racy like the swiftness of the current of the Ganges. His command of the English language and his style of writing attract the readers so much so that they cannot stop until they finish reading the whole text. Translating such a text is just like doing an acrobatic stunt with a sword. I went on translating it in such a way that the meaning of the source text is not lost and at the same time it fits into the beautiful idiom of the Telugu language.)

e) Kovvidi Lingaraju in the introduction to his Telugu translation of Maxim Gorky's Mother makes the following remark:

స్వతంత్ర రచనకన్నా అనువాదం చాలా కష్టం .అందులోనూ టాల్ స్టాయి, గోర్కీవంటి తత్వవేత్తల వుత్తమరచనలను తెలుగు చెయ్యడం మరీ కష్టం. గోర్కీ భాష అపూర్వమైనది. ఒక చిన్న వాక్యంలో మహత్తరమైన భావం యిమిడి వుంటుంది. అదీకాకుండా 
చదువుకొసే వాళ్ళు, తంత్ర రచనను మామూలు కొలతబద్ధతోటే కొలుస్తారు. కాని అనువాదాన్ని అసలు రచనను బట్టి కొలుస్తారు. అందుచేతనే విఖ్యాతులైన వాళ్ళ గ్రంధాన్ని అనువదించాలంటే యెంతో సమర్థత, సాహసం ఉండాలి. ఇల్టాంటి ఉత్తమమైన కల్పనను తెలుగు సోదరులు కూడా చదువుకోవాలసే కోరికే, నన్నీ సాహసానికి పురిగొల్పింది.

(Translating is much more complex than independent writing. It becomes even more difficult to translate the best works of philosophers like Tolstoy and Gorky. Gorky's language is extraordinary. A small sentence gets loaded with tons of meaning. In addition, the reader measures an independent writing in a normal way but when it comes to translation, the evaluation will be done in terms of the comparison with the original text. Thus, to translate the works of great writers, one has to be bold and requires a high degree of competence. It is the desire to make such great writings available to my Telugu brothers compelled me to undertake this adventurous task).

This kind of reverence shown to the source authors and their writings is in sharp contrast with what Edward Fitzgerald has stated in his translation of Omar Khayyam into English in a letter to his friend E.B.Cowell - "It is an amusement for me to take what liberties I like with these Persians who are not Poets enough to frighten one from such excursions, and who really do want a little art to shape them" (Fitzgerald, 1972, VI: xvi, quoted by Lefevere, 1990:12). 
We may also attribute it to the fact that in all these cases Telugu is the recipient, the target language which is translating from more established and well developed literatures, thereby introducing into its own culture and literature new genres, new models and new styles and new ideology. This practice helped Telugu not only in enriching its language and literature but also played a key role in strengthening its ties with these well-established literary traditions.

II. The various metaphors used by the Telugu translators to name their translations or to state what they think translation is for them are also interesting to note. Some of these metaphors are fresh, native and different from those that have sprung from the West:

a) Rayaprolu Subbarao in his translator's introduction to his Telugu translation-Ravindra Vyasavali came up with the following metaphor to state what translation is, rather not:

అనువాదమంటే ఒక కుండలో పాలు మరొక భాండములో పోసుకోవడం కాదు. ఒక్క ఆవును ఇద్దరు పిండుకోవడమూ కాదు. భాష యేదయినా కావచ్చు. మానవ హృదయమును అనునయించే కశ్చిదిప్టార్థ ప్రతీతి శబ్దవృత్తిలో లభిస్తున్నది-ఆది కవినీ కాలాన్నీ అధిగమించి ఉంటుంది-దానిని సంగ్రహించి అనుభవించడానికి జరిగే సారస్వత తంత్రణం) అనువాదం.

(Translation is not like simply transferring the milk from one pot into another. It is also not like two people milking one and the same cow. It can be any language. A quality that appeals to every human heart lies in its sound system. 
And it is something that goes beyond poets and ages. Translation is a literary exercise that tries to capture it and experience it.)

b) Y.C.P. Venkata Reddy, the translator of Kundanika Kapadiya's Gujarathi novel, Satpaglan Aakshman uses the popular metaphor

'అనువాదం కత్తిమీద సాములాంటిది'

(Translating is like doing an acrobatic stunt with a sword), which has also been used by many other translators like Jayashree Mohan Raj and R. Anantha Padmanabha Rao.

c) Nagnamuni in his introduction to an anthology of Telugu translations of Odia short story, Odia Kadhaa Sourabham by Upadrasta Anuradha states the following:

అసువాదం పెంకె గుర్రం లాంటిది. అశ్వహృదయం తెలిసిన రౌతుకు మాత్రమే లొంగుతుంది. లేకపోతే తిప్పి తిప్పి సేలకేసి విసిరికొడుతుంది. తిరిగి లేవకుండా ఎముకలు విరిగేటట్లు తుక్కు తుక్కుగా తొక్కేస్తుంది. పదహృదయం తెలియాలి. మాట మనసు ఎరగాలి.

అనువాదం మొసలిపై ప్రయాణం. క్షణక్షణం మారే నీటివాలు, వుధృతి, కరకర నమిలే భీకరమైన మొసలి కథలికలు తెలిసిన వారికి మాత్రమే మొసలి స్వారీ సాధ్య. అవతలి గట్టుకు చేరడం.

(Translation is like an untamed horse. It surrenders only to the rider who understands its heart. Otherwise, it twists and turns him round and round and hurls him to the 
H. Lakshmi

ground. And it stamps him very badly breaking his bones.

One has to know and understand the heart of the word.

Translation is like riding on a crocodile. The crocodile ride is possible only to those who know the direction of the flow of water, its force, and the movements of the ferocious crocodile, which can chew up the rider anytime. Only such riders would be able to reach the other shore.)

d) Jayashree Mohanraj in the introduction to her translation భారతీయ భాషా కథలు (Bhaaratiiya bhaasha kadhalu) published by Sahitya Akademy remarks as follows:

వాదం చేయడం కత్తిమీది సాము .అటు మూలరచన చేసిన రచయితలోకి పరకాయ ప్రవేశం చేసి భావాలను గ్రహిస్తూ యిటు లక్యభాషను చదివే పాఠకులను ఆకట్టుకుని అర్థమయ్యే భాషలో అనువాదం చేయాల్సి ఉంటుంది .ఇందులో ఎవరికి అన్యాయం చేసినా అనువాదం దెబ్బతింటుంది.

(Translating is like doing an acrobatic stunt with a sword. It is like the soul of the translator entering the body of the writer and grasping his innermost feelings and then translating them into the target language in the manner acceptable to the reader. Translation gets ruined if injustice is done to either of these things.)

It is interesting to observe the trope used here - పరకాయ ప్రవేశం (entering into another's body, in the context of translation it refers to the translator entering into the body of the source author). This is based on the Hindu philosophy known as 'Dwaita' which states that 
the body and the soul are two different things which can be separated.

III. The following are the different words used by the translators in the general sense of

Translation:

Anuvadam (Translation)

Anusrujana (Transcreation)

Racana (Writing)

Teligincu (Making it Telugu)

Tenigincu / Tenigincu (Making it Telugu)

Anukaranam (Imitation)

Andrikarana (Telugization)

Andrikruti (Transcreation in Telugu)

Andranuvadam (Telugu translation)

Telugu Anukruti (Telugu Transcreation)

Anumelana (Transcreation)

Tarjuma (Changing the form)

Tenuguseta (Making it Telugu)

Parivarthana (Mutation)

It can be observed from the above that in Telugu, except the three words 'Anuvadam' which is neutral, and the two wordsTarjuma and Parivarthana, which indicate changing over or transmutation, all the rest are more or less synonyms that indicate target-orientation and 'Telugization'. This itself speaks for the kind of translation activity that has taken place in the Telugu translation sphere and the norms that govern it.

IV. The following are some of the verbalizations regarding the process/nature of translation made either by the translators 
themselves or the experts who have written forewords or introductions to the translations:

a) Dasaradhi Rangacharya, a very famous writer and a translator who belongs to Telangana, in his introduction to the Telugu translation of the poetry of Mirza Ghalib from Urdu says the following regarding his process of translation:

అమరిపేటికలలో భద్రపరచబడిన మణిని సాధించడం ఎంత కష్టమో పద్యాలలో దాగిన గాలిబ్ హృదయాన్ని అందుకో వడం కూడా అంత శ్రమతో కూడిన పని. ప్రతి పద్యాన్నీ ఆకళించుకుని, ఆ పద్యానికి వివిధ వ్యాఖ్యాతలూ, విమర్శకులూ చెప్పిన అర్ధాన్ని చదివి నాకు స్ఫురించిన అర్ధాన్ని జీర్ణించుకుని, త్రేన్చి తెలుగు రూపంలో మళ్ళీ ఆ హృదయాన్ని ఆవిష్కరించడం కొంత క్లిష్టమైన పనే. ఒక్కొక్క పద్యాన్నీ తెలుగులోకి దింపడానికి రోజులు రోజులు పట్టేవి. అప్పటికీ సంపూర్ణంగా మూలార్ధం రాకుంటే మళ్ళీ మార్చవలసి వచ్చేది. ఇది అనువాదం కాని అనుసరణ కాదు. కనుక నా కల్పన ఈషణ్మాత్రమూ పనికిరాదు. తెలుగు పాఠకులకు గాలిబ్ భావం అందడానికి కావలసిన హంగులు చేయడానికి మాత్రమే నాకు అధికారం వుంది. అంతకు మించి లేదు. ఇలాటి నిర్టంధాలతో ఈ అనువాదానికి ఉపక్రమించాను.

ఈ పని) అనువాదం (చాలా కష్టసాధ్యమైనదనుటలో అతిశయోక్తి లేదు. ఒక్కొక్క పద్యాన్నీ జీర్ణించుకుని దాన్ని తెలుగులోకి దించడానికి రోజులు రోజులు పట్టేది. కడచిన1960 వ సంవత్సరమంతా గాలిబ్ కవితను మననం చేయడంలోనూ, దానిక 
తెనుగురూపం ఇవ్వడంలోనూ వ్యయించాను. ఆ సంవత్సరమంతా నా హృదయంలో సాక్షాత్కరించి అనుక్షణం నాకు తన హృదయ రహస్యములను బోధపరచిన అమరమూర్తి మీర్జా అసదుల్లాఖాన్ గాలిబ్ నా నర నరాలలో జీర్ణించుకుపోయినాడు. ఆయన అమృత హృదయం సర్వదా నా కవితకు క్లుప్తతను, గుప్తతను, ఆప్తతను ప్రసాదిస్తుందని ఆశిస్తున్నాను. బాషాభిమానులెల్లరు నా ఈ కృషిని సావధానంగా పరిశీలింతురు గాక!"

(Capturing the heart of Ghalib from his poems is as difficult as extracting the precious stone secured in a vault. Understanding the essence of every poem, reading the different interpretations of it given by different commentators and critics, and then digesting the meaning that occurs to me and belching it out to present the heart once again in the form of a Telugu poem, is indeed a complex task. I used to take days together to translate a single poem into Telugu. Even then, if the source meaning is not completely captured, I used to rewrite it again. This is a translation and not an adaptation. Even an iota of my own creation cannot be used. I have license only to make such modifications to the original that help make Ghalib's meaning comprehensible to the Telugu readers not more than that. I have embarked on this translation with all such constraints.

There is no exaggeration in saying that this work (translation) is a very difficult one. It used to take several days to digest each poem and compose it in Telugu. I have spent the whole year of 1960 in understanding Ghalib's poems and giving them a shape in Telugu. That whole year, Mirja Asadulla Ghalib resided in my 
H. Lakshmi

heart and explained the secrets of his heart every moment and thus became an integral part of every cell of my body. I hope his kind heart would gift my poetry with brevity, mystery and entrancement. May all the language lovers positively consider this effort of mine!')

In the same text, Devulappali Ramanuja Rao (the then Secretary, Andhra Pradesh Sahitya akademi) in his 'Avatharika' comes out with similar remarks as that of Dasaradhi regarding the process of translation. He too uses the word జీర్ణించుకొను (to digest). He also uses another word 'parivarthimcu'(to transform) for translation. Consider the following:

“శ్రి దాశరధి మహాకవి ఈ అనువాదమును మిక్కిలి ప్రతిభావంతముగా నిర్వహించినారు .అనువాద ధోరణి చాలా సరళంగానున్నది .అనువాదము కొరకు స్వీకరించిన ఛందస్సు , భాష ఔచిత్య శోభితములై యున్నవి .శ్రీ దాశరధి అనుసరించిన ఛందస్సు 'గజల్' పోకడకు అనుగుణముగా నున్నది . అనువాదకునికి మూల గ్రంధకర్తతో సమానమైన భావనాశక్తి అవసరమై యుండును .ఆ భావనాశక్తి శ్రీ దాశరధికి సంపూర్ణముగా కలదని ఈ అనువాదములోని ప్రతి పంక్తి నిరూపించుచున్నది . అనువాదము చేయుటలో శ్రీ దాశరధి చూపించిన నేర్పు అనస్యము.

గాలిబ్ కవిత్వములోని హైందవేతర వాతావరణమును హైందవ వాతావరణముగా పరివర్తించుటలో దాశరధి ప్రత్యేక ప్రతిభ చూపించినారు.

ఉమర్ ఖయ్యాంను జీర్ణించుకొని ఆంగ్లభాషలో పరివర్తించిన ఫిట్జుజీరాల్డువలె ,దాశరధి గాలిబ్ కవిత్వమును హృద్గతము 
గావించుకొని అనన్య సాధ్యమైన రీతిని ఆంధ్రీకరించినాడు . మూలమునకు అనువాదము కడు సరసముగా సాగినది . పూర్వజన్మలో దాశరధియే గాలిబేమో! లేక ఈ జన్మలో గాలిబే దాశరధియై అవతరించెనేమో!"

(Dasaradhi, a great poet, has done this translation in a very effective way. The translation reads very smooth. The language and prosody that are chosen for translation are very apt. The prosody adapted by Dasaradhi was appropriate to the 'Gazal' style. A translator requires the power of imagination equivalent to that of the source writer. Every line of this translation stands to prove that Dasaradhi has the kind of imaginative power-the skill that he displayed in translating this text is extraordinary!

Dasaradhi has exhibited a special talent in transforming the non-Hindu cultural context in Ghalib's poetry into that of Hindu.

Just like Fitzerald, who digested Omar Khayyam and transformed him into English, Dasaradhi got Ghalib's poetry melted into his heart and brought it out into the language of Andhra in an exceptional manner. Translation is very close to the source text. Probably, in the last birth Dasaradhi must have been Ghalib, or may be in this birth Ghalib has taken the form of Dasaradhi.

There are two points that are worth mentioning here. Firstly, as can be clearly observed from the above remarks, some resemblance to the notion of Cannibalism that emerged from the Brazilian school of Translation Studies. It basically refers to the idea of devouring the source text, digesting it and making it an integral part of your own self. This is an attempt to exploit the source texts in order to enrich the target culture and its literature. It is also to do with the dominant 
source and the dominated target culture. There is however a difference between the Brazilian context and the Indian context in general and the Telugu context in particular. Though both are postcolonial contexts, the Brazilian tendency of aggression and vengeance does not exist in the Telugu translation tradition. It is in this sense; it does not share with the Brazilian cannibalistic theory of translation, the postcolonial political attitude of the empire writing back, at least in the earlier times.

Secondly, the similarity with the sentiment expressed in many quarters in the field of translation- ' If Shakespeare were to write in Telugu, he would write it this way' or 'One wonders whether Shakespeare has written it in Telugu' and so on. Such remarks only point to the naturalness and readability of the translated texts in Telugu that would read as though they were originally written in Telugu. In other words the statements like పూర్వజన్మలో

\section{దాశరధియేగాలిబేమో! లేక ఈ జన్మలో గాలిబే దాశరధియై అవతరించెనేమో! (May}

be in the previous birth Dasaradhi himself was Ghalib! Or Ghalib himself was reborn as Dasaradhi in the present life!) would sound prima facie hyperbolic but reflect the sentiment related to the oneness of the source writer and the translator in terms of their creativity and literary genius. Yet another way of looking at it is from the perspective of dynamic equivalence proposed by Eugene Nida.

b) In the introduction to the Telugu translation of $\mathrm{C}$. Rajagopalachary's Tamil text-Srikrishnudu cuupina maargam (The path shown by Srikrishna), the publisherHindusamajam, makes the following remark: 
మరియొక భాష నుండి యనువదించవలసి

వచ్చినప్పుడునువాదకుడు రెండు విధములయిన భయములకు

లోనగును. మొదటిది మూల గ్రంథకర్త యభిప్రాయమునకు

భంగము కలుగునేమోయను భయము. రెడవది

మూలమునున్నదున్నట్లుగా, మక్కస్తముక్కగా

భాషాంతరీకరించిన యెడల అనువాదితీ గ్రంథమంత సుబోధకము

కాదేమోయను భయము.

(When it comes to translating from another language, the translator is always worried about two aspects. The first one is the fear that the source text might get distorted. The second one is that if a word to word translation is attempted, the translation may not be readable.)

This is the fear expressed by many translators all over the world. They intend to do justice to both the source text/author and the target audience. This is the reason why we say theoretically that the translators have to strike a balance between the two opposite poles-the source text and the target audience by going for a middle path. But it is easier said than done. Practically it may not be possible always. It goes without saying that a translation cannot afford to be too literal or too free. But as scholars working in the field of Translation Studies, we are now aware of the fact that there are many other things to be considered in a translation other than the source text and the target reader like the skopos of the translation, the ideology of the translator and the patron or the publisher, the 
translation norms that exist in the target culture at the given moment, the place occupied by the translation in the target polysystem and so on. It is of course unfair to expect these translators or reviewers to have the knowledge of the later developments in the field of Translation Studies and judge the translators going by them.

c) Rayaprolu Subbarao in his translator's introduction to his Telugu translation-Ravindra Vyasavali (1962) comments on the translation as follows:

కావ్య సృజనకు మూఖ్యాలంబనం శబ్దమే, అయినా అక్కడ శబ్దం బుజీషప్రాయమంటాడు. వొక వ్యాఖ్యాత. అంతకంటె శయ్యాగృహ కవాటంవంటి దంటే బాగుండేది. ఎందుకంటే వాక్యగతమైన శబ్దం చేతనంవలె వ్యవహరిస్తుంది. చల్లగాలి వీచిందంటీ తలుపు తెరుస్తుంది. చలిగాలి, అంటే మూసివేస్తుంది. రచనలో భావము ముత్యములో నీరులాగా ఇష్టార్థ లావణ్యముతో తొణికిసలాడుతూ వుంటుంది. కేవలం భావప్రకటన చేస్తే మీగడ తీసిన పెరుగు అవుతుంది. ాత్పర్యం వ్రాస్తే పొరిగింటి మజ్జిగలాగా పలచబడుతుంది. రచనలో శిల్పమునే కర్మకౌశలం వున్నది గదా! అందువల్ల అనువాదములో పదబంధమునూ, వాక్యరీతినీ కూడా వీలయినంతవరకూ సంగ్రహించడం ఆవశ్యక మనిపించింది.

(Form is central to the creation of any poetic work. But according to a critic the form is only accidental. I think it would be better if we say that form of a text is like a valve to the bed room. Because the form that constitutes a sentence works like a regulator. If a cool breeze comes, the 
window gets opened, and if the breeze is cold it gets closed. The sense expressed in a work shines gracefully like the water in a pearl. If only the meaning is expressed, it becomes like the curd after the cream is removed from it. If it is paraphrased, it gets diluted like the buttermilk borrowed from the neighbour. There is creativity and meaning in the form of the text itself and thus it is felt necessary that the collocations, the form, and the structure of the sentences should also be retained in the translation as far as possible.)

d) V. Venkatachalam in his English translation of monograph on Bhasudu (Telugu) by Pullela Sriramachandrudu remarks as follows regarding the translation:

ఆనువాదం మక్కీకి మక్కి కాకుండా ఉండేటట్లు ప్రయత్నించాను. అలాంటి అనువాదాలవల్ల మూలానికిగాని, ఏ బాషలోనికి అనువదిస్తామో ఆ భాషకు గాని ఏ మాత్రమూ ప్రయోజనం ఉండదు. నేను చేసిన అనువాదలన్నీ మూలంలోని అందాన్ని ఇంగ్లీషు అనువాదంలోనికి తీసుకొని వచ్చేటట్లు చేసిన స్వేచ్ఛానువాదాలు. శక్యమైనంత వరకు ఇవి మూలానికి దగ్గరగా ఉండేటట్లు చేసిన పునర్నిర్మాణాలు, కేవలం అనువాదాలు కావు.

(I tried not to go for word to word translation. Such translations are useful neither to the source language, nor to the target language. All my translations were free translations done in such a way that the aesthetic beauty of the source text was brought into English. These are reconstructions done to be as close as possible to the source text and are not mere translations.) 
e) M.B.S. Prasad in his introduction to Mullapudi Venkataramana's Telugu translations of Around the World in 80 Days' and PT 109: John F. Kennedy in World War II states the following:

రచయితలోకి పరకాయ ప్రవేశం చేసి ఆయన తెలుగులో రాసివుంటే ఎలా రాసేవాడో అలా రాశారు. తనే ఆ నవల ఒరిజనల్ రాసినంత ధాటీగా, స్వేచ్ఛగా రాశారు".

“ఈ నవల అనువాదం కూడా ఒరిజనల్ లాగానే గంభీరంగా సాగుతుంది."

“రెండు భాషలూ వచ్చినంత మాత్రాన అనువాదం చేయడం సాధ్యం కాదు. అనువాదంలో అందం, మూలానికి విధేయత - ఈ రెండింటిలోనూ ఏదో ఒకటే సాధ్యపడతాయంటారు పెద్దలు. అనువాదపు వాసనలు లేకుండా చక్కటి తెలుగు నుడికారంతో రాయగలగడం రమణకు అబ్బిన విద్య. ాలా విద్యలు నేర్వనిదే ఈ విద్య పట్టబడదు".

(This translation is done as if the translator's soul has entered into the source writer and the text is written in Telugu in such a way that had the source writer were to write it in Telugu he would have written in this way. Translation is done with such vigour and freedom as if the translator himself has written the original novel.)

The translation of this novel also reads as profound as the original. 
It is not possible to translate by simply knowing two languages. It is said that in any translation of the two things - beauty of the target text and fidelity to the source - only one is possible. Writing in a beautiful Telugu style using the native idiom without any features that mark it as a translation is a special skill that Ramana has acquired. It is not possible to master this skill unless one acquires many other skills.)

M.B.S. Prasad's above statement touches upon two thingsfirstly, how the translator has entered into the body of the source writer and has written this work as though the source writer himself is writing it in Telugu and secondly, the conflict between truth (fidelity) and beauty and how the translator has written the translation using fine Telugu idiom and taking care that the translation does not smell (read) like a translation.

\section{Conclusion:}

Coming to the norms of translation behavior in the Telugu context, one can see that the predominant view voiced by many translators, reviewers and scholars alike is that 'a translation has to read like something written originally in Telugu'. All such translations seem to have been considered as good/satisfactory translations by a vast majority of the Telugu reading public. One statement that keeps recurring in many reviews or forewords or introduction to translated literary works from other languages has been - 'This translation does not read like a translation. It reads as though it were originally written in Telugu'. This statement is used as a compliment, praising the work of a translator and is taken as sign of a good translation. This seems to be the predominant notion that the Telugu reading public have about a translation. Since they 
have been bred on the translations of the Mahabharat from Sanskrit into Telugu by the trio-Nannaya, Tikkana and Errapragada and several other subsequent translations from Sanskrit kavyas by other translators who have taken their predecessors as their role models, it is not surprising that they would want a translation to read like an original written in the target language and accepted as such despite their high regard and respect for the source writers. In a way the Telugu translation tradition seems to have always given priority to rewritings than to mere translations.

And some even use metaphors like 'parakaya pravesham' (entering into another body/soul, in this context the source writer's) and argue that the translator has to do this (in order to be a successful translator) or has done this; and some others strongly feel that the translator must have been the source author in his previous birth or the translation reads as though the source writer herself/himself has written it in Telugu. One does not know whether this kind of a praise showered on the translators by the experts is simply patting the back of the translator for all the hard work s/he has put in to make the source text available in the target language or really genuine and means what it says! Well, in many cases it could be former than the latter.

As far as the process of translation is concerned, all translators would state their dilemma overtly- how to remain faithful to the source writer/text and yet make the text readable in the target language. This conflict between loyalty to the source text and the readability of the target text, and the desire to balance both seem to mark the major concern of most of the translators. However, when one observes the actual practice of the translators, in a vast majority of the cases, irrespective of what they say in their introductions or 
translators' notes, what one realizes is that in their actual practice they give more preference to the readability of the target text than the fidelity to the source and would sacrifice the former for the sake of the latter. One can thus conclude that it is the readability of the target text and a desire to make it read as though it is originally written in Telugu seems to have been the unwritten norm of translation that played a prescriptive role and informed the translation process and thus ultimately had a greater role in shaping the nature of translations in the Telugu translation tradition until very recent times when the new crop of academics started taking the practice of translation seriously and would foreignize their translations giving priority to the source text and its cultural, stylistic and literary nuances rather than the convenience of the target readers. It is especially the case with the recent translations made from Indian languages into English. It is interesting to note that even now we find two categories of translations that are being published in the Indian context in general- the scholarly and the public, and each of these not only have their own specific market but also earmarked function.

\section{Note:}

1. All English translations are by me unless otherwise mentioned.

\section{References:}

Anantha Padmanabha Rao, R. (1992). Morning Face (Telugu Translations of Mulkraj Ananad's novel), New Delhi: Sahitya Academy.

Anjaneya Sharma, V. (1969). Andra Padya Gitanjali (Telugu Tr.) Tirupati: Sri Venkateshwara University. 
Anuradha, Upadrasta. (2008). Oriya Kadha Saurabham (a Telugu translation of Oriya short stories), Vijayawada: Cinuku Pracuranalu.

Dasaradhi, Rangacharya. (1961). Ghalib Gitaalu (A Telugu translation of Mirza Ghalib's Urdu Poetry), Machilipatnam: M.Seshachalam \& Co.

Dwa.Naa.Sastry. (1998). Telugu Sahitya Charitra, Hyderabad: Vishalandra Publishing House.

Genette, Gerard. (1997). Paratexts: Thresholds of Interpretation, Cambridge University Press.

Jayashree, Mohanraj. (2012). Bharatiya Bhashaa Kadhalu (19002000) (Telugu translation of the anthology of short stories edited by EVR), Bangalore: Sahitya Academy.

Kovvidi, Lingaraju. (1981). Mother (Telugu translation of Maxim Gorky's novel by the same name), Hyderabad: Vishalandhra Publishing House.

Lefevere, Andre. (ed.). (1990). Translation History Culture: A Sourcebook, London and New York: Routledge.

Lakshmikanta Mohan, M. (1961). Lear Raju (A Telugu Translation of Shakespeare's Play King Lear), Hyderabad: Sri Saraswathi Book Depo.

Munday, Jeremy. (2001). Introducing Translation Studies, London and New York: Routledge.

Narayana Rao, Cilukuri, (1940). Sri Krishnudu Cuupina Maargam (A Telugu translation of C.Rajagopala Chary's Tamil text), Rajamandry: Hindu Samajam. 
Pingali, Lakshmi Kantham. (1974). Andhra Sahitya Charitra, Hyderabad: Andhra Pradesh Sahitya Academy.

Sreeramachandrudu, P. (1991). Bhasudu (A Telugu translation of the monograph in English on Bhasudu by Venkatachalam, V), New Delhi: Sahitya Academy.

Subbarao, Rayaprolu. (1962). Ravindra Vyasavali (Telugu Tr.), New Delhi: Sahitya Academy.

Suri, Tenneti.(1986). Rendu Mahanagaraalu (Telugu translation of Charles Dickens' A Tale Of Two Cities), Hyderabad: Vishalandra Publishing House.

Toury, Gideon. (1978/2000). "The Nature and Role of Norms in Literary Translation" In Venuti Lawrence (ed.) Translation studies, 2000.pp.198- 211.

Toury, Gideon. (1980). In Search of a Theory of Translation, Tel Aviv: The Porter Institute for Poetics and semiotics, Tel Aviv University.

Toury, Gideon. (1995). Descriptive Translation Studies and Beyond, Amsterdom and Philadelphia: John Benjamins.

Venkata Ramana, Mullapudi.(2006). 80 rojullo bhupradakshinam\& Dwitiya prapamca samgramamlo Kenadi (Telugu translations), Vijayawada: Vishalandra Publishing House.

Venkata Reddy, Y.C.P. (2000). Satpaglan Aakshman (A Telugu translation of Kundanika Kapadiya's Gujarathi novel by the same name), New Delhi: Sahitya Academy.

Venuti, Lawrence, (2000). Translation Studies, London and New York: Routledge. 